about minimizing differences in economic outcome from country to country.

EPL: What do you think are the major strengths of environmental policy in the Netherlands and what would you view as the priorities for specific action in the next few years?

Ginjaar: We have in this country as in most countries a situation in which we are still developing environmental law and the instruments to conduct environmental policy. We have a law for air pollution and a law against the pollution of surface water; both of these are already in effect. But we also have a law on waste and chemical waste which we are now trying very hard to put into effect. A law against noise hindrance is presently in Parliament; the next step is to translate it into action and that will take 7 to 10 years. A law on the protection of soil is in preparation now, as well as the law on chemicals and the law of what we call external safety of industries.

I think three problems are most im. portant for any environmental policy in this country. The first is to harmonize the laws that we have and those that are in preparation. Because they were drawn up at different times, under different conditions and with different insights, there are a lot of differences. We are trying to harmonize them into one large framework so they are all operating along the same lines. Connected with this is the harmonization of the responsibilities of the lower governmental bodies, the local and provincial governments, particularly as regards licencing authority. I think this is a very large problem with a very high priority.

The second problem that we have is the financing of environmental measures. The polluter-pays principle says that the man who is causing the pollution has to pay for the protection measures. Well, that occurs in the normal day to day life of society. But government administration costs must also be paid, particularly the costs to local government bodies. According to the polluter-pays principle, we can achieve that only by levies or charges - a highly political topic. The question that must be resolved is should these costs be paid by special charges or by general taxation.

The third topic is the integration of environmental policy with total government policy. I feel that it is not sufficient to have laws against air pollution, water pollution, and so on. Govern- ment policies should be such that from the beginning environmental issues play a role in the decision process. I think that for the next 10 or 20 years, the most important issue is this integration of environmental policy at all levels of government policy. And I think that the environmental impact statement and the setting of standards are most important in accomplishing this.

\title{
BOOK NOTES
}

Heribert Rausch: Die Umweltschutzgesetzgebung. Aufgabe, geltendes Recht und Konzepte.

\section{Schulthess Polygraphischer Verlag, Zürich 1977. 284 pp.}

L'étude est une "publication d'habilitation" à la Faculté de Science Juridique de Zurich, ce qui correspond à une thèse de professorat. L'auteur s'engage dans une nouvelle voie pour localiser le droit de la protection de l'eenvironnement d'une manière systématique. Rausch procède d'abord à l'analyse du droit ancien et actuel de la protection de l'environnement Suisse. Après cette mise au point, il avance des arguments qui surtout grâce à la comparaison juridique au 3ème chapitre, tracent des pensées originales susceptibles d'animer les programmes internationaux de l'environnement.

Après la démonstration de la fonction de la protection de l'environnement sur cinq modèles de pensée, Rausch se penche sur le concept dogmatique du droit de la protection de l'environnement et arrive à la conclusion qu'une base de règlement unitaire de la protection de l'environnement (p.e. sous forme de définition) ne peut guère être déterminée d'une manière satisfaisante.

Dans la 2ème partie de l'étude, l'auteur donne un excellent résumé de la réglementation de la protection de l'environnement actuellement en vigueur en Suisse. Il pose la question de la classification juridique de l'article 24 septies de la constitution fédérale soit en tant que règle de compétence, soit règle de programme ou même comme droit fondamental. Tout compte fait Rausch reconnaît que ce règlement revêt un caractère similaire au droit fondamental. Il constate que ceci crée une obligation plus grande qu'avant pour les organes législatifs en vue de rendre plus efficace la législation en matière de protection de l'environnement. Ce sont d'ailleurs les derniers efforts de l'Institut pour une Politique Européenne de 1'Environnement qui reflètent ces problèmes de la raison d'être de la garantie constitutionnelle et à caractère de droit fondamental au profit de l'individu d'un environnement sain et plus équilibré.

Dans le 3ème chapitre, l'auteur développe, en guise d'une comparaison juridique intense, une série de concepts stratégiques du droit de l'environnement. Voici à titre d'exemple les concepts suivants: la dilution et la concentration, la meilleure technologie, les circulations fermées, le recensement juridique de la conduite individuelle (la responsabilité civile et le droit pénal inclus) l'internalisation des charges (Internalisirung der Kosten), délimitation de l'accroissement, le règlement des valeurs de la qualité de l'environnement et le principe de la persévérance. L'auteur souligne à très juste titre que les différents concepts se croisent et qu'ils devraient se compléter dans l'application. Du point de vue systématique, le concept de la fixation des valeurs de la qualité de l'environnement devrait être mis au point d'urgence par les sciences naturelles à leur tour. Le concept mentionné, de même que le principe de la persévérance, doivent remplir d'ailleurs une fonction d'ordre téléologique, alors que les autres concepts servent principalement d'aide de décision. C'est une idée bien originale, que d'envisager une fois l'analyse du droit de l'environnement par les objectifs stratégiques et non seulement à travers les institutions existantes de l'environnement.

En résumé nous sommes en présence d'une étude fort bien documentée et digne d'être lue par chaque juriste de l'environnement. L'ouvrage devrait également animer d'autres Etats à procéder à une comparaison juridique. De cette manière, la discussion sur le droit de l'environnement pourrait être essentiellement enrichie aussi sur le plan des programmes internationaux.

Dr. Alfred Rest, Université Cologne 